\title{
Embolização Arterial Seletiva no Tratamento de Volumoso Quisto Ósseo Aneurismático da Bacia
}

\author{
Selective Arterial Embolization for a Large Pelvic Aneurysmal Bone Cyst \\ Treatment
}

Joaquim SOARES DO BRITO ${ }^{1}$, José PORTELA ${ }^{1}$

Acta Med Port 2015 Nov-Dec;28(6):780-783

RESUMO

O quisto ósseo aneurismático corresponde a uma lesão tumoral benigna do aparelho locomotor em idade jovem, que pode adquirir um comportamento biológico agressivo. Estas lesões necessitam frequentemente tratamento cirúrgico através de curetagem e preenchimento com enxerto ósseo, no entanto, em lesões com maior agressividade está indicada a resseção tumoral em bloco. Lesões agressivas apresentam habitualmente grandes dimensões e por vezes são difíceis de abordar cirurgicamente pela localização e necessidade de reconstrução complexa. Neste contexto, a embolização arterial seletiva surge como possibilidade terapêutica adjuvante ou mesmo definitiva. Este trabalho relata um caso paradigmático da utilização da embolização arterial seletiva no tratamento de um volumoso quisto ósseo aneurismático de difícil abordagem cirúrgica.

Palavras-chave: Embolização Terapêutica; Ossos Pélvicos; Quistos Ósseos Aneurismáticos.

\section{ABSTRACT}

Aneurysmal bone cysts are benign musculoskeletal tumours, which occur in young patients and, occasionally, with aggressive behaviour. Those tumours very often will need surgical treatment with curettage and bone grafting, but for aggressive lesions, in bloc resection is more advisable. Aggressive tumours use to be large and sometimes with difficult surgical approach and demanding complex surgical reconstructions. In this scenario, selective arterial embolization is a possible primary or adjuvant treatment option. This paper presents a paradigmatic case, where selective arterial embolization allowed a successful treatment of a large aneurysmal bone cyst with difficult surgical resolution.

Keywords: Bone Cysts, Aneurysmal; Embolization, Therapeutic; Pelvic Bones.

\section{INTRODUÇÃO}

O quisto ósseo aneurismático corresponde a uma lesão tumoral benigna do aparelho locomotor, osteolítica e hemorrágica, que pode adquirir um comportamento biológico agressivo com expansão e destruição óssea. ${ }^{1-4} \mathrm{~A}$ maioria destas lesões tumorais surge em doentes jovens (1 ${ }^{\mathrm{a}} / 2^{\mathrm{a}}$ décadas de vida), distribuindo-se por várias localizações, como os ossos longos do membro inferior, superior e coluna verteral. ${ }^{1}$ A localização na bacia é menos comum, podendo variar entre os $8 \%$ e $12 \%{ }^{4-7}$ Raramente, podemos observar quistos ósseos aneurismáticos que curam de forma espontânea, no entanto, a grande maioria necessita tratamento cirúrgico. ${ }^{5,8}$

O comportamento biológico benigno destas lesões permite que sejam preferencialmente aplicadas técnicas cirúrgicas de curetagem e preenchimento com enxerto ósseo, no entanto, em lesões de maior agressividade está indicada a resseção em bloco. ${ }^{5}$ Estas lesões mais agressivas, habitualmente de grandes dimensões e difíceis de abordar cirurgicamente pela localização e necessidade de reconstrução complexa, levaram ao desenvolvimento de técnicas adjuvantes (ou até mesmo para tratamento primário) como a crioterapia, injeção intralesional de agentes esclerosantes ou embolização arterial seletiva, melhorando a taxa de sucesso no tratamento das lesões, minorando as taxas de recidiva local. . $^{57,9,10}$

Este artigo descreve o caso clinico de um doente do género masculino com 19 anos de idade e volumoso quisto ósseo aneurismático localizado sobre o ilíaco direito, tratado com sucesso através de embolização arterial seletiva.

\section{CASO CLÍNICO}

Doente do género masculino, 19 anos de idade, leucodérmico, referenciado em junho de 2010 para consulta de ortopedia por dor e impotência funcional da anca direita, com quatro semanas de evolução, sem história traumática

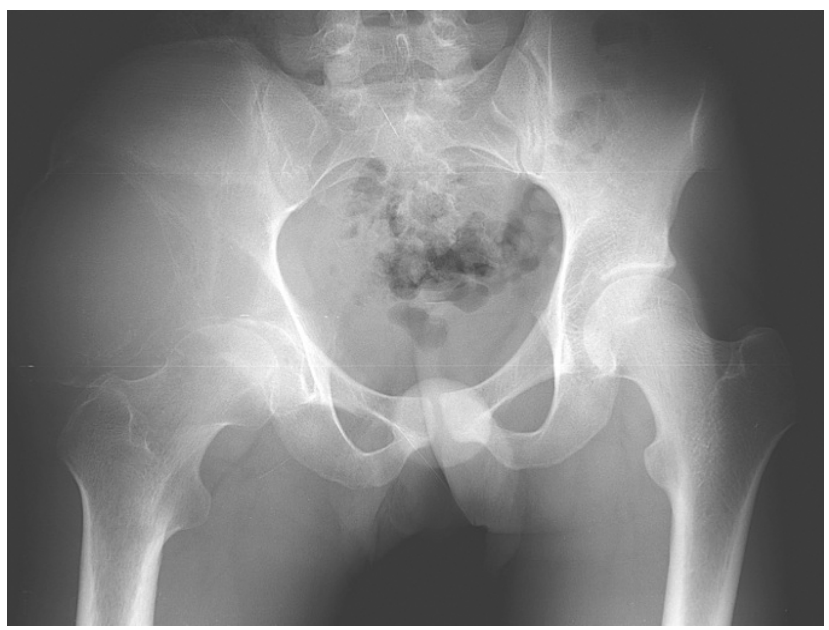

Figura 1 - Radiografia simples da bacia com imagem de lesão expansiva sobre o ilíaco direito

\footnotetext{
1. Serviço de Ortopedia e Traumatologia. Hospital de Santa Maria. Centro Hospitalar Lisboa Norte. Lisboa. Portugal.

$\triangle$ Autor correspondente: Joaquim Soares do Brito. joaquimsoaresdobrito@gmail.com

Recebido: 14 de Julho de 2015 - Aceite: 14 de Outubro de 2015 | Copyright (C) Ordem dos Médicos 2015
} 
ou outra sintomatologia relevante associada.

A avaliação clinica objetivou dor na mobilidade articular da anca direita e grande limitação funcional. Foi requisitada radiografia da bacia (Fig. 1), que levantou suspeita de lesão expansiva localizada ao ilíaco direito. Neste contexto foram adicionalmente requisitados tomografia computorizada (Fig. 2), cintigrama e biopsia guiada por imagem, que foram compatíveis com diagnóstico de quisto ósseo aneurismático.

Perante o diagnóstico obtido, tendo em conta a grande dimensão da lesão e a abordagem cirúrgica previsivelmente complexa, decidiu-se realizar a embolização arterial seletiva da mesma, que decorreu no serviço de Imagiologia da nossa instituição (Fig. 3). Em decúbito dorsal e sob anestesia geral balanceada, foi cateterizada a artéria femoral esquerda e com auxilio do intensificador de imagem detetados os vasos nutritivos da lesão tumoral sobre o ilíaco direito. Após identificação dos referidos vasos procedeu-se à sua embolização com biosferas que decorreu sem complicações. A duração do procedimento foi de aproximadamente quatro horas. O doente seguiu para a unidade de recobro pós-anestésico onde permaneceu durante algumas horas em vigilância, tendo alta clinica no próprio dia. $O$ doente foi posteriormente seguido em consulta externa.

Às duas semanas pós-embolização objetivou-se dor residual moderada, e anemia aguda no contexto do efeito roubo gerado pela embolização da enorme lesão hemangiomatosa. Aos três meses de evolução, o doente apresentava anca e joelho em flexo, mas com radiografia demonstrando maior calcificação da massa óssea aneurismática (Fig. 4). Aos seis meses pós-embolização constatou-se ausência de queixas álgicas, melhoria funcional evidente com resolução do flexo, e radiografia com calcificação global da lesão. A evolução manteve-se clinica e radiologicamente favorável sem registo de outras intercorrências durante o período de seguimento.

Aos três anos pós-embolização o doente mantém dor residual, apresentando capacidade para desempenhar atividades de vida diária sem dificuldade e prática desportiva recreativa ocasional (Fig. 5). Objetivamente apresentava-se com mobilidade da anca melhorada, realizando $70^{\circ}$ de flexão anterior, extensão de $-10^{\circ}$, rotação externa de $15^{\circ}$, rotação interna de $0^{\circ}$, abdução de $70^{\circ}$ e adução de $15^{\circ}$.

\section{DISCUSSÃO}

Quistos ósseos aneurismáticos são frequentemente encontrados nas regiões metafisárias dos ossos longos dos membros inferior e superior, assim como na coluna vertebral, no entanto, a sua localização sobre a bacia é menos frequente. ${ }^{11}$ As lesões agressivas constituem um desafio no seu tratamento adequado, principalmente quando se encontram sobre regiões anatómicas de difícil acesso. ${ }^{11}$
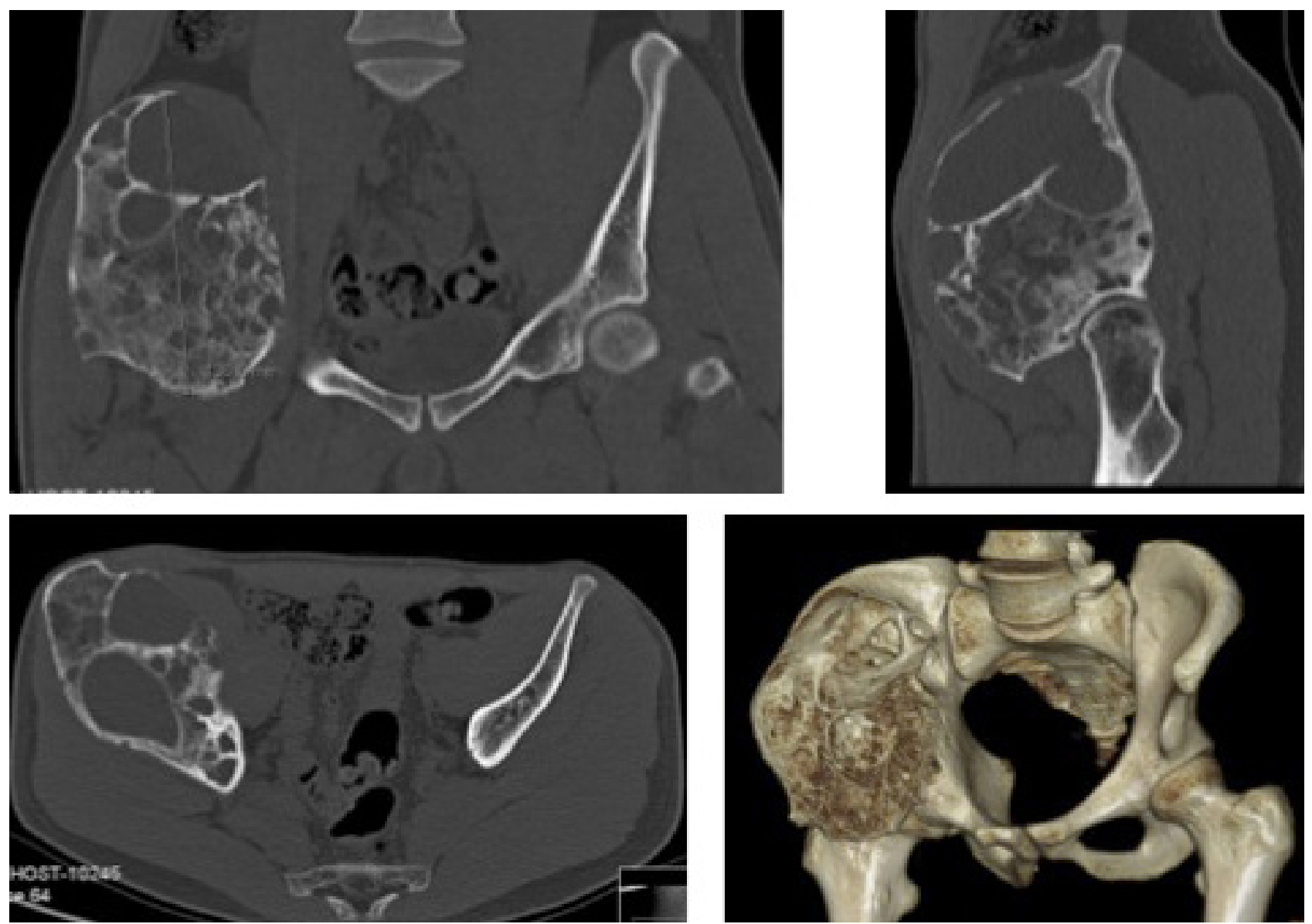

Figura 2 - Imagens de tomografia computorizada da cavidade pélvica onde é visível lesão expansiva de grandes dimensões sobre o ilíaco direito 

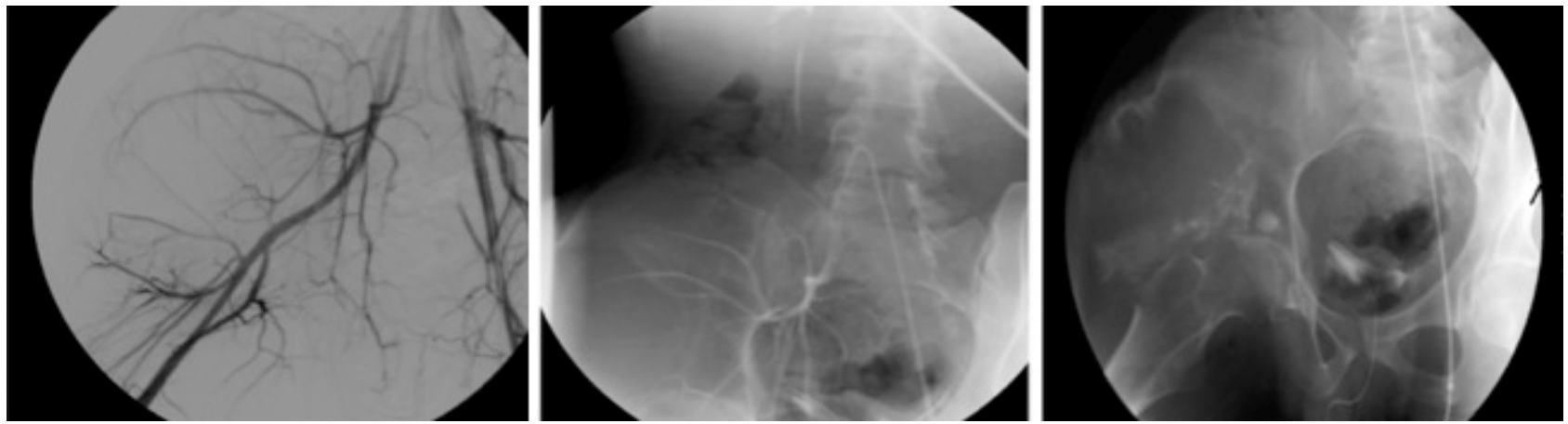

Figura 3 - Embolização arterial seletiva do quisto ósseo aneurismático

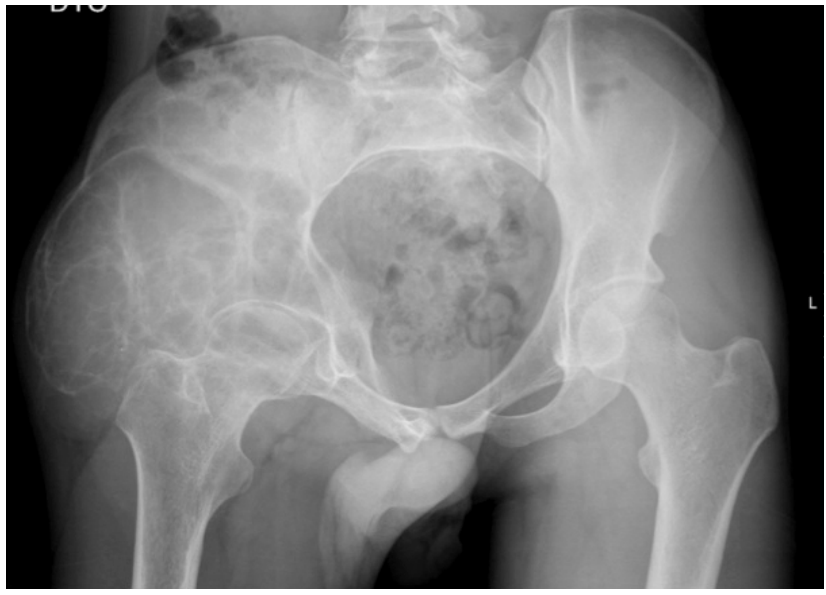

Figura 4 - Radiografia simples da bacia aos 3 meses pós-embolização demonstrando calcificação da massa aneurismática

Deste modo, as opções terapêuticas deverão ser cuidadosamente equacionadas, tendo em conta não só a localização da lesão, como a sua dimensão, vascularização e relação com os tecidos adjacentes. ${ }^{5,6,11}$ É igualmente fundamental ter em conta os principais diagnósticos diferenciais destas lesões tumorais, como é o caso do tumor de células gigantes, e principalmente o osteosarcoma telangiectásico. Deste modo, a biópsia adquire grande importância na determinação do diagnóstico e consequentemente do tratamento a realizar. ${ }^{1}$

Lesões com dimensões inferiores a $5 \mathrm{~cm}$ de maior diâmetro e que exibam destruição óssea mínima, pouca expansão cortical e que não afetem a integridade articular do acetábulo ou articulação sacroilíaca, são habitualmente tratadas com curetagem e enxerto ósseo. ${ }^{6}$ Por outro lado, lesões com dimensões acima de $5 \mathrm{~cm}$ de maior diâmetro ou com evidência de maior agressividade traduzida pela destruição óssea e expansão da cortical, requerem técnicas cirúrgicas mais agressivas que podem incluir a resseção em bloco, no entanto, a resseção completa é difícil em algumas localizações como é o caso da bacia. ${ }^{6} \mathrm{~A}$ embolização arterial seletiva tem sido, neste contexto, indicada como tratamento adjuvante ou mesmo definitivo. ${ }^{12}$

A grande vantagem da embolização arterial seletiva é evitar a cirurgia e, deste modo, as complicações peri-operatórias que estão inerentes e associadas a procedimentos reconstrutivos de elevada complexidade. ${ }^{5} \mathrm{O}$ sucesso deste procedimento para quistos ósseos aneurismáticos é variá-

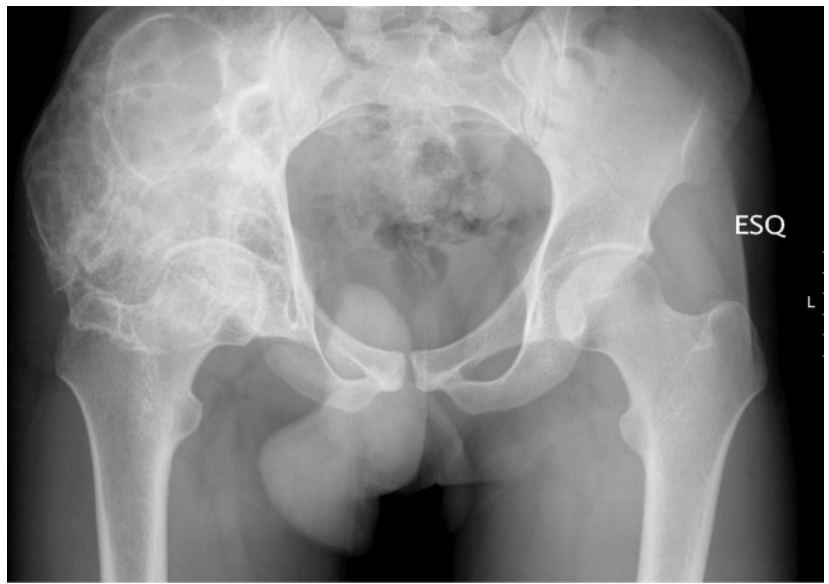

Figura 5 - Radiografia simples da bacia aos 3 anos pós-embolização

vel, oscilando entre $75 \%$ e $94 \% .{ }^{13}$

A taxa de recidiva descrita após a realização deste tipo de procedimento pode atingir números tão elevados como $44 \%$, relacionando-se particularmente com os quistos de dimensão acima de $5 \mathrm{~cm} .{ }^{10-14}$ No entanto, e perante uma recidiva é sempre possível realizar embolizações seriadas. ${ }^{5,10}$ Como todos os procedimentos, a embolização também acarreta riscos, estando a taxa de complicações situada em redor dos $6 \% .{ }^{5,10,13,14}$ Constituem complicações desta técnica a disseção da artéria femoral, dor secundária à necrose isquémica do tumor, embolização inadvertida de vasos responsáveis pela vascularização de território não tumoral, infeção, síndrome pós-embolização, entre muitas outras com menor expressão. 5,10,13,14 A embolização de vasos nutritivos de território não tumoral pode constituir uma complicação particularmente grave, com lesões cutâneas, necrose muscular e risco acrescido pela isquémica associada. ${ }^{5}$ De modo a prevenir estas complicações é fundamental esta técnica ser realizada por um especialista experiente, capaz de avaliar cuidadosamente os vasos nutritivos da lesão e de embolizar seletivamente as artérias patológicas, escolhendo para este fim o agente embólico mais adequado. 5,14

\section{CONCLUSÃo}

Em conclusão, o tratamento dos quistos ósseos aneurismáticos deverá ser cuidadosamente equacionado, considerando a idade do doente, dimensão da lesão, 
agressividade e localização. Neste artigo é relatado o sucesso terapêutico de um volumoso quisto ósseo aneurismático de difícil acesso cirúrgico, através de uma única embolização arterial seletiva e sem complicações significativas associadas. Este caso constitui um exemplo da validade desta técnica, que na indicação correta e com aplicação adequada pode ser uma mais-valia no tratamento deste tipo de lesões tumorais.

\section{PROTECÇÃO DE PESSOAS E ANIMAIS}

Os autores declaram que os procedimentos seguidos estavam de acordo com os regulamentos estabelecidos pelos responsáveis da Comissão de Investigação Clínica

\section{REFERÊNCIAS}

1. Campanacci M, Bertoni F, Bacchini P. Aneurysmal bone cyst. In: Campanacci M. Bone and soft tissue tumors. Berlin: Springer; 1990. p. 725-51.

2. Vergel De Dios AM, Bond JR, Shives TC, McLeod RA, Unni KK. Aneurysmal bone cyst: a clinicopathologic study of 238 cases. Cancer. 1992;69:2921-31

3. Campanacci M, Capanna R, Picci P. Unicameral and aneurysmal bone cysts. Clin Orthop Relat Res. 1986;204:25-36.

4. Capanna R, Bertoni F, Bettelli G, Present D, Biaginil R, Ruggieri P, et al. Aneurysmal bone cysts of the pelvis. Arch Orthop Trauma Surg. 1986;105:279-84.

5. Rossi G, Mavrogenis AF, Papagelopoulos PJ, Rimondi E, Ruggieri P. Successful treatment of aggressive aneurysmal bone cyst of the pelvis with serial embolization. Orthopedics. 2012;35:e963-8.

6. Yildirim E, Ersözlü S, Kirbaş I, Özgür AF, Akkaya T, Karadeli E. Treatment of pelvic aneurysmal bone cysts in two children: selective arterial embolization as an adjunct to curettage and bone grafting. Diagn Interv Radiol. 2007;13:49-52.

7. Papagelopoulos PJ, Choudhury SN, Frassica FJ, Bond JR, Unni KK, Sim FH. Treatment of aneurysmal bone cysts of the pelvis and sacrum. e Ética e de acordo com a Declaração de Helsínquia da Associação Médica Mundial.

\section{CONFIDENCIALIDADE DOS DADOS}

Os autores declaram ter seguido os protocolos do seu centro de trabalho acerca da publicação dos dados de doentes.

\section{CONFLITOS DE INTERESSE}

Não existem conflitos de interesse a declarar.

\section{FONTES DE FINANCIAMENTO}

Não foi recebido qualquer subsídio ou bolsa.

J Bone Joint Surg Am. 2001;8:1674-68.

8. Malghem J, Maldague B, Esselinckx W, Noël H, De Nayer P, Vincent A. Spontaneous healing of aneurysmal bone cysts. A report of three cases. J Bone Joint Surg Br. 1989;71:645-50.

9. Rossi G, Mavrogenis AF, Rimondi E, Ciccarese F, Tranfaglia, C, Angelelli $B$, et al. Selective arterial embolisation for bone tumours: experience of 454 cases. Radiol Med. 2011;116:793-808.

10. Rossi G, Rimondi E, Bartalena T, Gerardi A, Alberghini M, Staals EL, et al. Selective arterial embolization of 36 aneurysmal bone cysts of the skeleton with N-2-butyl cyanoacrylate. Skeletal Radiol. 2010;39:161-7.

11. Kim CG, Kweon SH. Primary aneurysmal bone cyst in the iliac bone: a case report. Hip Pelvis. 2014;26:202-5.

12. Mohit AA, Eskridge J, Ellenbogen R, Shaffrey $\mathrm{Cl}$. Aneurysmal bone cyst of the atlas: successful treatment through selective arterial embolization: case report. Neurosurgery. 2004;55:982.

13. Mavrogenis AF, Rossi G, Rimondi E, Papagelopoulos PJ, Ruggieri P. Embolization of bone tumors. Orthopedics. 2011;34:303-10.

14. Green JA, Bellemore MC, Marsden FW. Embolization in the treatment of aneurysmal bone cysts. J Pediatr Orthop. 1997;17:440-3. 


\section{Embolização Arterial Seletiva no Tratamento de Volumoso Quisto Ósseo Aneurismático da Bacia}

Acta Med Port 2015:28:780-783

Publicado pela Acta Médica Portuguesa, a Revista Científica da Ordem dos Médicos

Av. Almirante Gago Coutinho, 151

1749-084 Lisboa, Portugal.

Tel: +351218428215

E-mail: submissao@actamedicaportuguesa.com

www.actamedicaportuguesa.com

ISSN:0870-399X | e-ISSN: 1646-0758

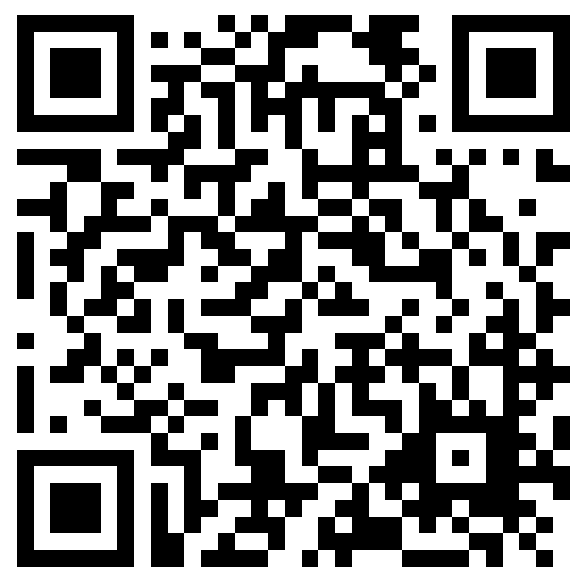

